\title{
Cardiac troponin I and T: Exploring popularity with Google Trends
}

\author{
Giuseppe Lippi ${ }^{1}\left(\mathbb{D}\right.$, Fabian Sanchis-Gomar ${ }^{2}$ \\ ${ }^{1}$ Section of Clinical Biochemistry, University of Verona, Italy \\ ${ }^{2}$ Department of Physiology, Faculty of Medicine, University of Valencia \\ and INCLIVA Biomedical Research Institute, Valencia, Spain
}

There is now firm evidence that the measurement of cardiac troponins, either I (cTnI) or T (cTnT) using high-sensitivity immunoassays, is the mainstay for diagnosing both ischemic and non-ischemic myocardial injury, regardless of the cutoff point, as we have read in the article by Bjurman et al. [1]. Recent evidence attests that neither of these two biomarkers seem superior to the other for the purposes of diagnosing myocardial infarction, so that they can be ideally used interchangeably [2] Since little information is currently available on the worldwide preference of one biomarker over the other, a retrospective analysis was carried out based on Google Trends, to establish which of the two cardiac troponins is more popular than the other.

Google Trends (Google Inc., Mountain View, CA, USA) is one of the most widely accessed tools in digital epidemiology. It is a freely available Google instrument, typically used for analyzing the popularity of top search queries in Google over time, across various geographical regions and using different languages. The search volume (expressed as Google Trends weekly Score) is finally displayed in graphical format, where the top value (i.e., 100) represents the maximum volume of Google searches for one or more given keywords [3]. In the present analysis, we used the keywords "troponin I" and "troponin T" in the field "topic", with no geographical or language restriction (thus including potential local translations), from the oldest searchable period (i.e., year 2004) up to present time (i.e., January 2020). The results of this search were downloaded in comma-separated values, and were imported into an Excel file (Microsoft, Redmond, WA, USA), and analyzed with Analyse-it
(Analyse-it Software Ltd, Leeds, UK). Results were expressed as mean \pm standard deviation and significance of differences was assessed with the Mann-Whitney test. The study was performed in accordance with the Declaration of Helsinki, under the terms of relevant local legislation.

The primary results of weekly volume of Google searches for cTnI and cTnT from 2004 to the present year are shown in Figure 1. Although the volume of weekly Google searches for cTnI and $\mathrm{cTn} T$ was found to be almost identical between the years $2004-2010(49.3 \pm 18.2$ vs. $49.4 \pm 11.2$; $\mathrm{p}=0.478$ ), the number of weekly searches in Google for cTnT became steadily higher than that for $\mathrm{cTnI}$ in the following period, i.e., between 2011 to present year $(57.0 \pm 12.1$ vs. $42.5 \pm 8.7$; mean difference, $29 \%$ and $95 \%$ confidence interval [CI] $27-31 \%$; $p<0.001)$. This trend was magnified during the prior 12 months, whereby comparing the weekly volume of Google searches for cTnT $(80.7 \pm 4.1)$ and $\mathrm{cTnI}(58.8 \pm 3.8)$, the mean gap further increased to $31 \%$ (95\% CI 27-35\%; $\mathrm{p}<0.001)$. Interestingly, the weekly volume of Google searches for cTnI remained higher in United States, Mexico, Brazil, Portugal, France, Iran, China, and Japan, amongst others, while that for cTnT appeared to be predominant in the remaining countries.

It has recently been demonstrated that assessment of Web-based inclinations, using tools such as Google Trends, reflects human behaviors along with preference to access some specific diagnostic and/or therapeutic resources [4], including the worldwide use of cardiac biomarkers [5]. Therefore, the results of this analysis suggests that, despite the existence of only one fully-automated

Address for correspondence: Prof. Giuseppe Lippi, Section of Clinical Biochemistry, University Hospital of Verona, Piazzale LA Scuro, 37134 Verona, Italy, tel: +39-045-8124308, fax: +39-045-8122970, e-mail: giuseppe.lippi@univr.it 


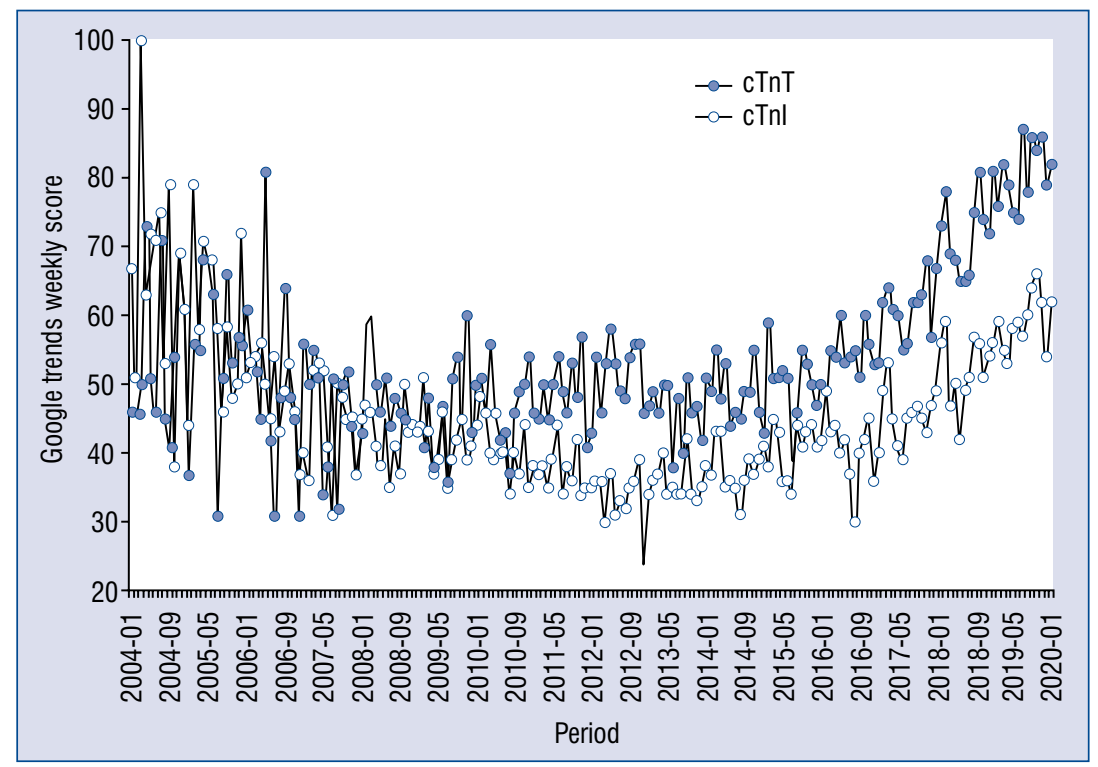

Figure 1. Trend of worldwide weekly Google Trends searches for cardiac troponin I (cTnl) and cardiac troponin T (cTnT) between 2004 and January 2020.

immunoassay for measuring cTnT, the current popularity of cardiac biomarkers seems to favor cTnT. Even in areas traditionally bound to cTnI, such as Canada and northern Europe, the scenario has changed during the past decade, especially during the past 12 months, with a weekly volume of Google searches for cTnT offsetting that for cTnI. The recent clearance of high-sensitivity-cTnT immunoassay in United States is probably linked with the observed change in tendency, which has led to broad diffusion of the test in the country [6]. Interestingly, the gap between the weekly Google searches for cTnI and cTnT in the United States has narrowed from $\sim 50$ vs. $\sim 20$ in the mid-2010s, to $\sim 50$ vs. $\sim 35$ in the past 12 months (data not shown).

\section{Conflict of interest: None declared}

\section{References}

1. Bjurman C, Zywczyk M, Lindahl B, et al. Decreased admissions and hospital costs with a neutral effect on mortality following lowering of the troponin T cutoff point to the 99th percentile. Cardiol J. 2017; 24(6): 612-622, doi: 10.5603/CJ.a2017.0079, indexed in Pubmed: 28695975.

2. Lippi G, Cervellin G. Is one cardiac troponin better than the other? J Lab Precis Med. 2019; 4: 19, doi: 10.21037/jlpm.2019.04.06.

3. Lippi G, Mattiuzzi C, Cervellin G. Is digital epidemiology the future of clinical epidemiology? J Epidemiol Glob Health. 2019; 9(2): 146, doi: 10.2991/jegh.k.190314.003, indexed in Pubmed: 31241874.

4. Mavragani A, Ochoa G, Tsagarakis KP. Assessing the methods, tools, and statistical approaches in google trends research: systematic review. J Med Internet Res. 2018; 20(11): e270, doi: 10.2196/jmir.9366, indexed in Pubmed: 30401664.

5. Lippi G, Mattiuzzi C, Cervellin G. Trends of popularity of cardiac biomarkers: Insights from Google Trends. Emergency Care Journal. 2018; 14(3): 7769, doi: 10.4081/ecj.2018.7769.

6. Rubini Gimenez M, Badertscher P, Twerenbold R, et al. Impact of the US food and drug administration-approved sex-specific cutoff values for high-sensitivity cardiac troponin $\mathrm{T}$ to diagnose myocardial infarction. Circulation. 2018; 137(17): 1867-1869, doi: 10.1161/ CIRCULATIONAHA.117.031940, indexed in Pubmed: 29685935. 